\title{
Leonurine Preconditioning Attenuates Ischemic Acute Kidney Injury in Rats by Promoting Nrf2 Nuclear Translocation and Suppressing TLR4/ NF- $\kappa$ B Pathway
}

\author{
Li Han, ${ }^{a}$ Aimei Chen, ${ }^{a}$ Ling Liu, ${ }^{a}$ and Fang Wang $*, b$ \\ ${ }^{a}$ Department of Traditional Chinese Medicine, Lianyungang TCM Branch of Jiangsu Union Technical Institute; \\ Jiangsu 222007, China: and ${ }^{b}$ Department of Thyroid Disease, Hubei Provincial Hospital of Traditional Chinese \\ Medicine; Hubei 430074, China. \\ Received August 28, 2021; accepted October 15, 2021
}

Despite the precise mechanisms for renal ischemia/reperfusion (I/R)-induced acute kidney injury (AKI) are poorly understood, nuclear factor erythroid 2 related factor 2 (Nrf2) and Toll-like receptor 4 (TLR4) pathways were considered as the important targets. Leonurine (LEO) is a special alkaloid extracted from Chinese motherwort (Leonurus japonicus Houtt), which has an anti-inflammatory effect and reduces oxidative stress. We conducted the study to explore the efficacy of LEO against I/R-induced AKI in rats and further investigated the underlying mechanisms. Ischemic renal injury was induced by temporary vascular clamping for $45 \mathrm{~min}$. We have measured the levels of inflammation-related biomarkers and antioxidative stress markers. Next, Western blot analysis and Real-time PCR were performed to analyze whether the Nrf2 and TLR4/nuclear factor-kappaB (NF- $\kappa \mathrm{B})$ pathways were involved in this process. We found that LEO pretreatment remarkably decreased serum creatinine and blood urea nitrogen (BUN) in I/R rats and attenuated acute tubular damage. In addition, LEO markedly increased the expression of antioxidant proteins and decreased the levels of inflammatory factors. Further study revealed that LEO promoted Nrf2 into the nucleus, promoted the expression of heme oxygenase-1 (HO-1) and quinone oxidoreductase 1 (NQO-1), and suppressed the TLR4/NF- $\kappa$ B signal pathway in kidney tissues of ischemic AKI rats. The study reveals that LEO has a protective effect to prevent ischemic AKI through activation of Nrf2 nuclear translocation resisting oxidative stress injury and inhibition of the TLR4/NF- $\kappa$ B pathway mediated inflammatory gene expression.

Key words Leonurine; acute kidney injury; nuclear factor erythroid 2 related factor 2; Toll-like receptor 4

\section{Introduction}

As we all know, acute kidney injury (AKI) is a common clinical problem characterized by a sudden deterioration in renal function within $7 \mathrm{~d}$, leading to electrolyte imbalance and acid-base imbalance, resulting in high morbidity and mortality. There are many causes of AKI, including ischemia, hypoxia, and nephrotoxicity. ${ }^{1)}$ The primary cause is ischemiareperfusion (I/R) injury, resulting from trauma, shock, sepsis, and renal transplantation. ${ }^{2)}$ Apart from renal replacement therapy, ${ }^{3)}$ supportive care, and palliative care, there is no targeted pharmacologic therapy is effective in patients with severe I/R-induced AKI. Therefore, new therapeutic strategies that preserving renal impairment and maximizing recovery of renal function after I/R-induced AKI are urgently needed.

The inflammatory response and oxidative stress are critical events in I/R-induced AKI, which set off many harmful events that may promote additional damage to the kidney. ${ }^{4,5}$ It is well established that Nuclear factor erythroid 2 related factor 2 (Nrf2) signaling pathway regulates oxidative stress by increasing gene expression of a series of antioxidants. $\left.{ }^{6}\right)$ After cellular insult, the Nrf2 nuclear translocation promotes heme oxygenase-1 (HO-1) and reduced nicotinamide adenine dinucleotide phosphate (NADPH): quinone oxidoreductase 1 (NQO-1) expression, which encodes antioxidants involved in cellular protection and the antioxidant effects. ${ }^{7,8)}$ Accumulating pieces of evidence indicated that directly or indirectly reducing the inflammation response and oxidative stress is of great significance to alleviating I/R-induced AKI. After kidney ischemia, a large number of endogenous ligands are released from damaged renal parenchymal cells and bind to Toll-like receptor 4 (TLR4). ${ }^{9)}$ TLR4 activates the downstream nuclear factor $-\kappa \mathrm{B}(\mathrm{NF}-\kappa \mathrm{B})$, promotes NF- $\kappa \mathrm{B}$ protein to enter the nucleus, affects the transcription of genes, stimulates a variety of inflammatory cytokine expression, and exacerbating the process of I/R-induced AKI.

Leonurine (LEO) is a special alkaloid, which is found in Chinese motherwort (Leonurus japonicus Houtt). Numerous studies have shown that LEO exerts multiple biologic effects, including protective effects on ischemic myocardium and brain, ${ }^{10,11)}$ antioxidative, ${ }^{12)}$ and anti-inflammatory. ${ }^{13)}$ Song et al. reported that LEO has an anti-inflammatory effect by inhibiting the expression of the TLR 4 pathway in mammary glands of mice mastitis model. ${ }^{13)}$ Chen et al. found that LEO reduces oxidative stress in aging mice via activating the $\mathrm{Nrf} 2$ pathway. ${ }^{14)}$ However, whether the LEO has a protective role on ischemic AKI is still largely unknown. The purpose of this study was to assess the roles of LEO pretreatment on I/R rats and to demonstrate its relevant molecular mechanisms.

\section{Experimental}

Chemical and Animal LEO (CAS: 24697-74-3, shown in Fig. 1A) were purchased from Aladdin (Beijing, China) and dissolved in a saline solution. Animal experiments in this study were approved by Hubei University of Traditional Chinese Medicine Institutional Animal Care and Use Committee. Healthy adult male Sprague-Dawley (SD) rats were 
A<smiles>COc1cc(C(=O)OCCCCN=C(N)N)cc(OC)c1O</smiles>

B

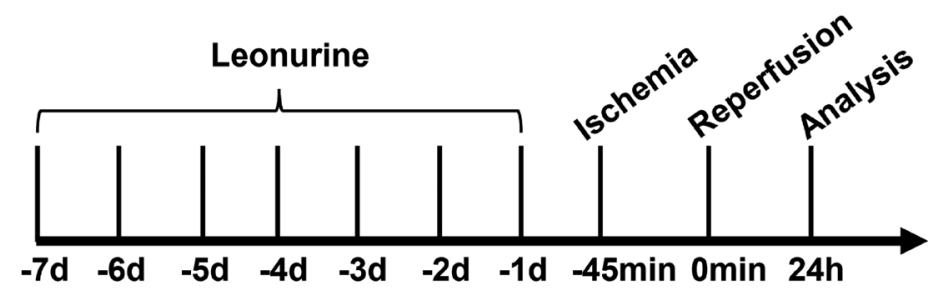

C

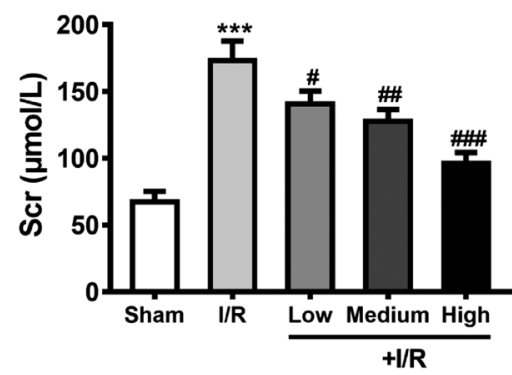

D

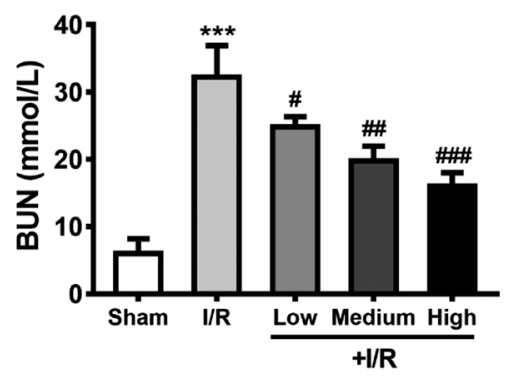

E

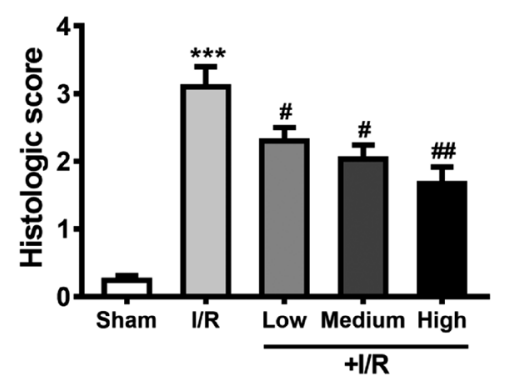

F
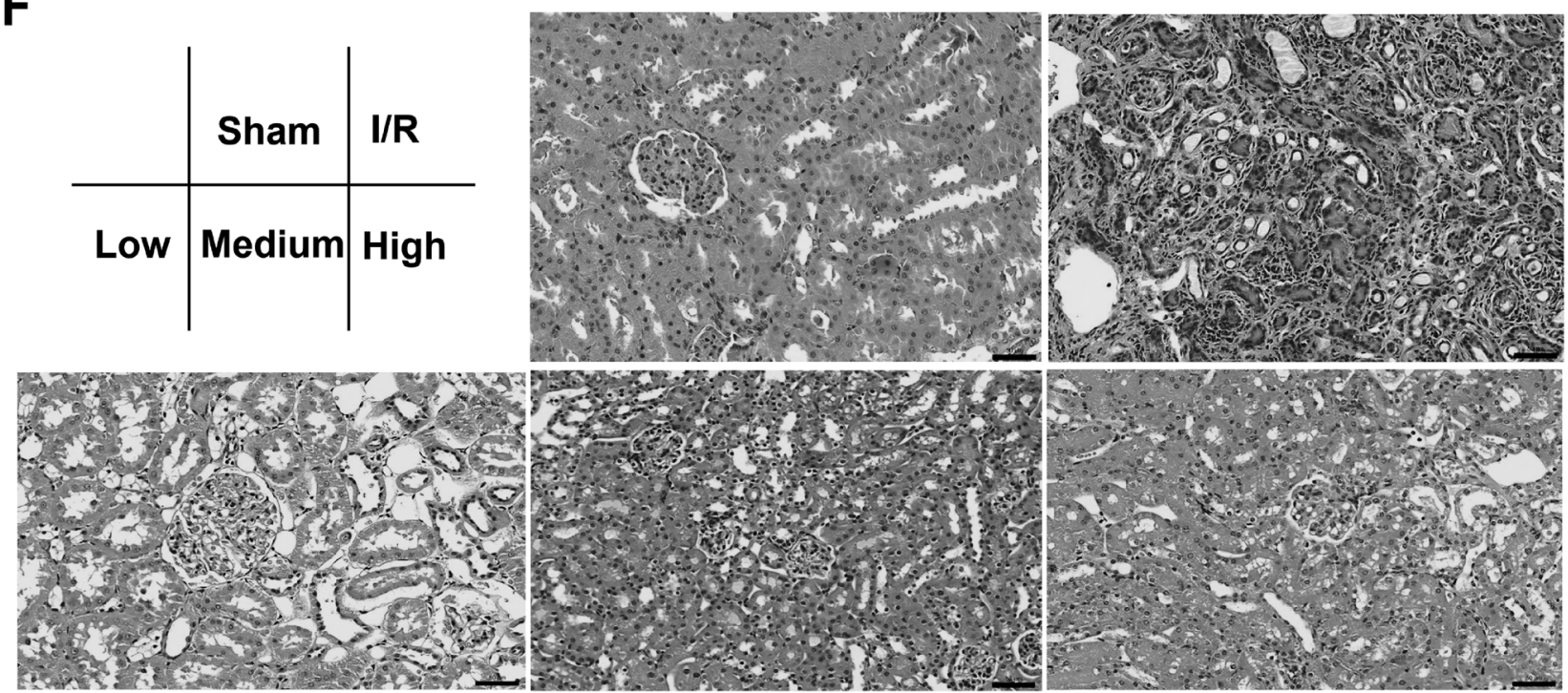

Fig. 1. Preconditioning of LEO Alleviates Renal Dysfunction and Histopathology in I/R Rat Model

(A) Chemical structure of LEO. (B) Flow chart of the animal experimental design. SD rats were given LEO gavage suspension orally for one week before I/R kidney damage. (C) Serum creatinine levels. Low, Medium, and High: low (7.5 mg/kg), medium (15 mg/kg), and high (30 mg/kg) doses of LEO. (D) BUN levels. (E) The histologic score was performed by a semi-quantitative method. (F) Representative images of H\&E staining of kidney tissue slices $(200 \times$ magnification, scale bar $=50 \mu$ m). Values are given in the form of mean \pm S.D. ( $n=6$ rats in each group). ${ }^{* * *} p<0.001$ versus sham; ${ }^{\#} p<0.05,{ }^{\# \#} p<0.01,{ }^{\# \#} p<0.001$ versus $\mathrm{I} / \mathrm{R}$.

acquired at Vital River Laboratories (Beijing, China). The rats were maintained in an specific-pathogen-free (SPF) building (temperatures of 21 to $24^{\circ} \mathrm{C}$; relative humidity between 40 and $55 \%$ ) with an appropriate day/night cycle. During feeding, all rats had unrestricted access to food and water.

Animal Model and Experimental Therapy We performed a renal $\mathrm{I} / \mathrm{R}$ injury model in rats based on previously reported studies ${ }^{15)}$ after a week of adaptation. The rats were given anesthesia with $1 \%$ pentobarbital sodium $(50 \mathrm{mg} / \mathrm{kg}$, Sigma-Aldrich, MO, U.S.A.) by intraperitoneal injection. Once exposed, hemostasis microvascular clamps were placed on both sides of renal pedicles to occlude for $45 \mathrm{~min}$. Following the desired period of ischemia, the clamps were then released and observed visually the normal supply of blood flow. Rats in sham group went through all same surgical steps except for clamping the renal pedicles. We randomly divided 30 rats into five groups, as described below: (1) sham group and I/R group, pretreated with normal saline for $7 \mathrm{~d}$, (2) low-dose group, pretreated with LEO $(7.5 \mathrm{mg} / \mathrm{kg} / \mathrm{d})$, (3) Medium-dose group, pretreated with LEO $(15 \mathrm{mg} / \mathrm{kg} / \mathrm{d})$, and (4) High-dose group, pretreated with LEO $(30 \mathrm{mg} / \mathrm{kg} / \mathrm{d})$. The dosage of LEO used in the experiment refers to previous literature reports ${ }^{16}$ ) and the experimental design is schematically represented in (Fig. 1B).

Evaluation of Renal Function Abdominal aorta blood was taken from rats in each experimental group following 
$24 \mathrm{~h}$ reperfusion. Renal function was monitored by measuring the amount of Serum creatinine (Scr) and blood urea nitrogen (BUN) in different groups with commercially available kits (Mindray Bio-Medical, Shenzhen, China) according to the reagent instructions.

Histologic Examination The kidney tissues were obtained at the end of reperfusion to evaluate the histopathological changes. The tissues were conventionally fixed by $10 \%$ formaldehyde, paraffin-embedded, and sectioned. After hematoxylin and eosin (H\&E) staining, kidney tissues were visualized by an optical microscope to analyze pathological changes. Renal injury scores of each group were calculated by two researchers in a blinded fashion and scored using a semiquantitative pathological scoring system as described previously. The grade of renal tissue damage was scored ranging from 0 (normal kidney) to 4 (severe damage). ${ }^{17}$ )

Cytokines Detection The blood samples of each group were centrifuged at $3000 \times \boldsymbol{g}$ for $30 \mathrm{~min}$ and the serum was transferred in aliquots into new centrifuge tubes. The levels of inflammatory cytokines: interleukin-1 $\beta$ (IL-1 $\beta$ ), tumor necrosis factor $\alpha$ (TNF- $\alpha$ ), IL-6, and IL- 8 in serum were determined by enzyme-linked immunosorbent assay (ELISA) kits (Boster, Wuhan, China), following instructions and the absorbance of the samples were read at $450 \mathrm{~nm}$ using an ELISA plate reader (type Elx800, Bio-Tek Instruments, VT, U.S.A.).

Measurement of Oxidative Stress Markers Oxidative stress markers such as superoxide dismutase (SOD), glutathione peroxidase (GSH), catalase (CAT), and malondialdehyde (MDA) in the kidney homogenate were detected by the corresponding assay kits (NJJC Bio, Nanjing, China) following the supplied instructions. In brief, the activity of SOD, GSH, and CAT was assessed respectively using the absorption of 550, 412 , and $240 \mathrm{~nm}$, and the MDA level was calculated based on the absorption of $450 \mathrm{~nm}$. In addition, protein content in different groups was determined by Bradford reagents (Beyotime, Jiangsu, China).

Western Blotting We extract total, separate cytoplasmic, and nuclear proteins from the kidney tissues using the radio immunoprecipitation assay (RIPA) lysis buffer, NE-PER Cytoplasmic, and Nuclear Extraction Reagents (Thermo Scientific, CA, U.S.A.) following the steps of the kit instructions. Added protein loading buffer $(5 \times$, Beyotime, Jiangsu, China) in a ratio of $1: 5$ to protein sample, and denatured the mixture for $30 \mathrm{~min}$ at $95^{\circ} \mathrm{C}$. Protein samples $(12 \mu \mathrm{g}$ lane-1) were separated by a sodium dodecyl sulfate-polyacrylamide gel electrophoresis (SDS-PAGE) and transferred to a polyvinylidene fluoride membrane (Millipore Immobilon, Darmstadt, Germany). After blocking, the membranes were incubated with corresponding primary and secondary antibodies. These primary antibodies diluted at 1:1000: anti-glyceraldehyde-3-phosphate dehydrogenase (GAPDH) (Beyotime), anti-PCNA (Beyotime), anti-Nrf2 (Cell Signaling Technology, MA, U.S.A.), anti-HO-1 (Boster), and anti-NQO-1 (Boster), anti-TLR4 (Beyotime), anti-MyD88 (Boster), anti-NF- $\kappa$ B p65 (Boster), anti-Phospho-NF- $\kappa$ B p65 (Boster), anti-inhibitor kappaB-alpha $(\mathrm{I} \kappa \mathrm{B} \alpha)$ (Beyotime), anti-Phospho- $\mathrm{I} \kappa \mathrm{B} \alpha$ (Beyotime). Protein signals were detected using electro-chemiluminescence (ECL) chemiluminescence reagent (Thermo Scientific). The relative optical densities of proteins were assessed using Image $\mathrm{J}$ software (NIH, MD, U.S.A.).

Real-Time Quantitative PCR Total RNA was obtained
Table 1. Primer Sequences

\begin{tabular}{lll}
\hline \hline Gene name & Primer & \multicolumn{1}{c}{ Sequence $5^{\prime}>3^{\prime}$} \\
\hline \multirow{2}{*}{ GAPDH } & Forward & AAGACCCAGAAATGAAC \\
& Reverse & TCTACACGATAACAACCA \\
\multirow{2}{*}{ HO-1 } & Forward & CCATAGGCTCCTTCCTCCTTTC \\
& Reverse & GGCCTTCTTTCTAGAGAGGGAATT \\
\multirow{2}{*}{ NQO-1 } & Forward & TCACCACTCTACTTTGCTCCAA \\
& Reverse & TTTTCTGCTCCTCTTGAACCTC \\
\hline
\end{tabular}

from kidney homogenate (TaKaRa Bio, Otsu, Japan), then total RNA was reverse-transcribed into cDNA using a ReverTra Ace RT kit (Toyobo, Dalian, China), and quantitative PCR was performed using an SYBR Ex Taq kit (TaKaRa Bio). GAPDH was served as a reference gene. Gene expression differences were calculated using the $2^{-\Delta \Delta \mathrm{Ct}}$ method. The primer sequences used in this study were shown in Table 1.

Statistical Analysis All results were produced as the means \pm standard deviations (S.D.) and based on measurements from at least three independent experiments. All the statistical analysis was done using the GraphPad Prism version 7.0 for Windows (GraphPad Software, CA, U.S.A.). The significance of differences among experimental groups was analyzed using Student's $t$-test or one-way ANOVA. A $p$-value of $<0.05$ was defined as statistically significant.

\section{Results}

Protective Effects of LEO Pretreatment on Rats with Ischemic AKI Here, we detected renal function and histological changes in different groups. As shown in (Figs. 1C, 1D), the levels of serum $\mathrm{Cr}$ and BUN, which are markers of renal function, were measured. I/R group showed significant increases in $\mathrm{Cr}$ and BUN against the sham group. LEO pretreatment prevented $\mathrm{Cr}$ and BUN increase, this effect was more obvious in the $30 \mathrm{mg} / \mathrm{kg}$ group. Subsequently, kidney tissues from the different groups were stained with H\&E to observed pathologic examination and evaluate the severity of damage with a semi-quantitative scoring (Figs. 1E, 1F). In the sham group, we did not find specific structure abnormalities, while I/R induced visible pathological changes in the kidney, such as cell swelling, blebbing, and inflammatory cell infiltration. These structural modifications of kidneys improved in rats pretreated with low, medium, and high doses of LEO before I/R injury. Furthermore, compared to the I/R group, the histologic score of the three LEO groups was significantly reduced. Overall, these data demonstrates that LEO prevented renal dysfunction and histologic damage in ischemic AKI.

LEO Reduced Oxidative Stress in Kidney Tissues from Ischemic AKI Rats From literature, oxidative damage is closely related to ischemic AKI. ${ }^{18)}$ Therefore, we evaluated the effects of LEO on oxidative stress by determining MDA content and the activities of SOD, CAT, and GSH enzymes in the kidney tissue homogenate of rats in each group. As illustrated in Fig. 2, MDA levels in I/R rats showed a significant increase, while SOD, CAT, and GSH activities decreased significantly. However, MDA levels in the LEO groups which pretreated with high-dose, medium-dose, and low-dose LEO were significantly decreased, whereas the activity of SOD, CAT, and GSH was significantly increased. Our results show that LEO can attenuate oxidative stress in ischemic AKI rats.

LEO Inhibited Cytokines Production of Ischemic AKI 
A

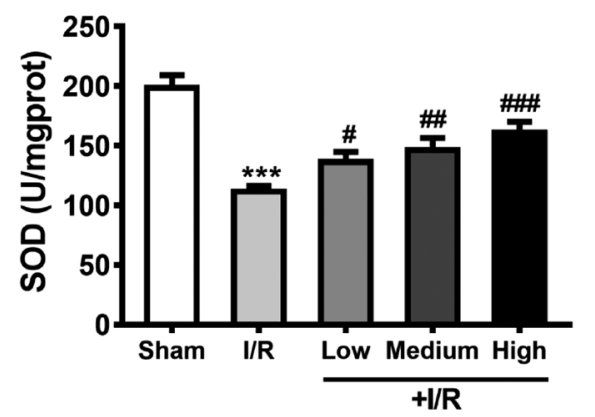

C

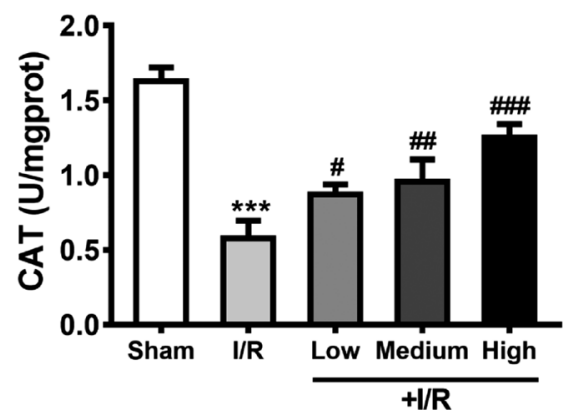

B

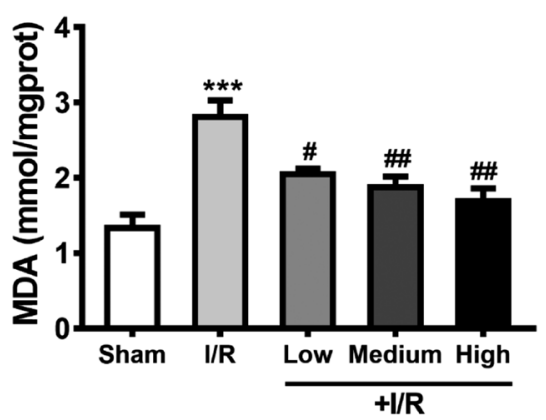

D

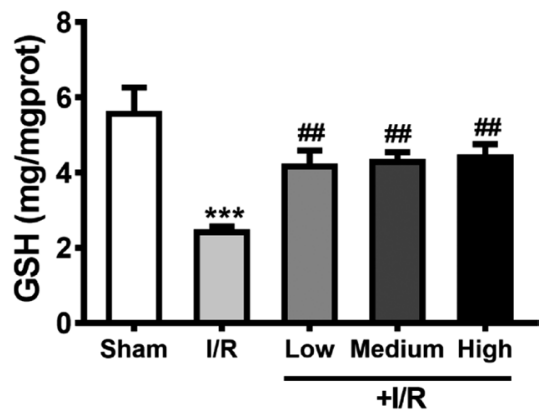

Fig. 2. LEO Reduced Oxidative Stress in Kidney Homogenate from Ischemic AKI Rats

SOD (A), CAT (C) and GSH (D) activity and MDA (B) content in tissue homogenate were analyzed using corresponding reagents. Values were given in the form of mean \pm S.D. $\left(n=6\right.$ rats in each group. $* * * p<0.001 ;{ }^{\#} p<0.05,{ }^{\# \#} p<0.01$, and ${ }^{\# \# \#} p<0.001$ versus $\mathrm{I} / \mathrm{R}$.

A

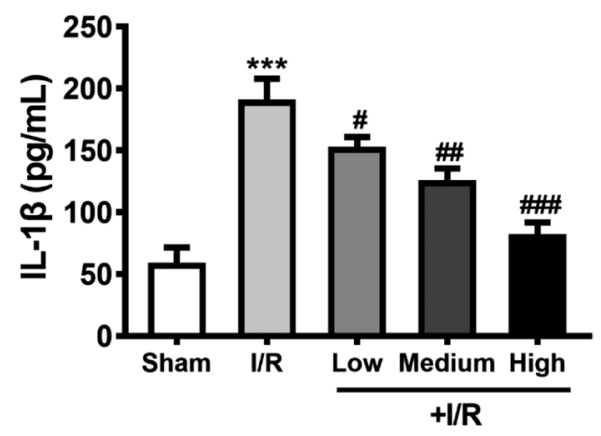

C

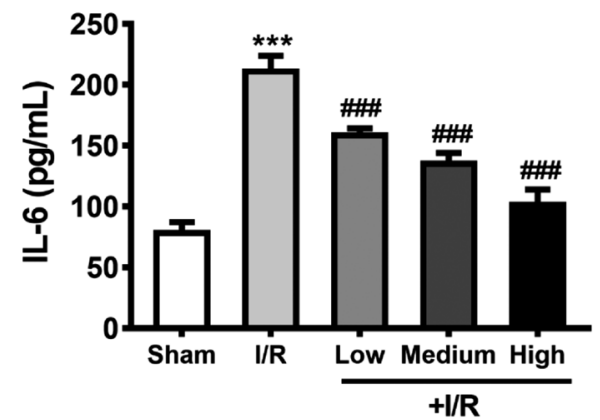

B

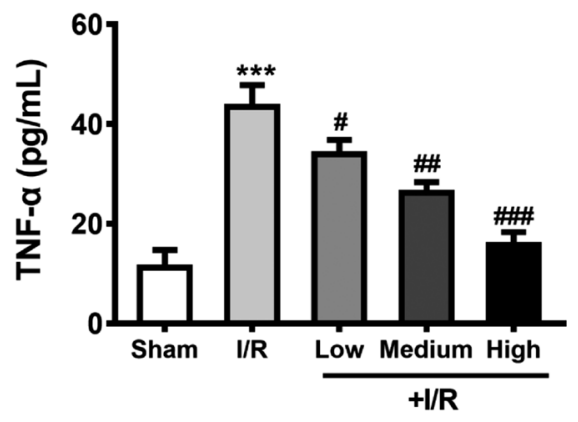

D

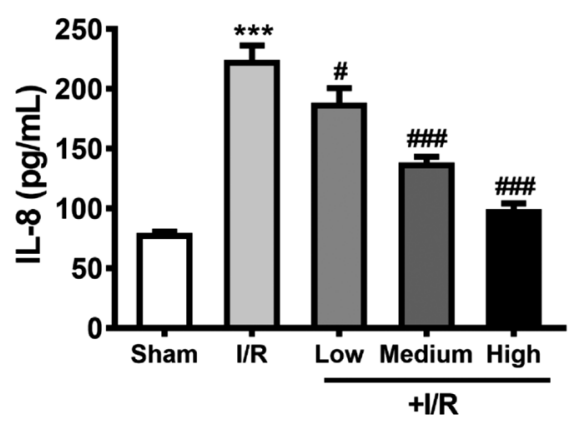

Fig. 3. LEO Decreased the Level of Cytokine in Ischemic AKI

Quantitation of IL-1 $\beta$ (A), TNF- $\alpha$ (B), IL-6 (C), and IL-8 (D) in serum was performed by ELISA. Data were expressed as mean \pm S.D. $(n=6$ rats each group). $* p<0.05$ versus sham; ${ }^{\#} p<0.05,{ }^{\# \#} p<0.01$ versus $\mathrm{I} / \mathrm{R}$, *** $p<0.001$ versus sham; ${ }^{\# \#} p<0.001$ versus $\mathrm{I} / \mathrm{R}$. 
Rats We observed serum levels of four inflammatory cytokines (Fig. 3) after reperfusion in various groups. I/R induced an increase in content in IL- $1 \beta$, TNF- $\alpha$, IL-6, and IL- 8 compared with those of the Sham group. However, for the
I/R group, LEO significantly reduced the levels of the four previously mentioned inflammatory factors following $I / R$. The above findings suggest that LEO alleviates the production of inflammatory cytokines in rats following $\mathrm{I} / \mathrm{R}$ injury.

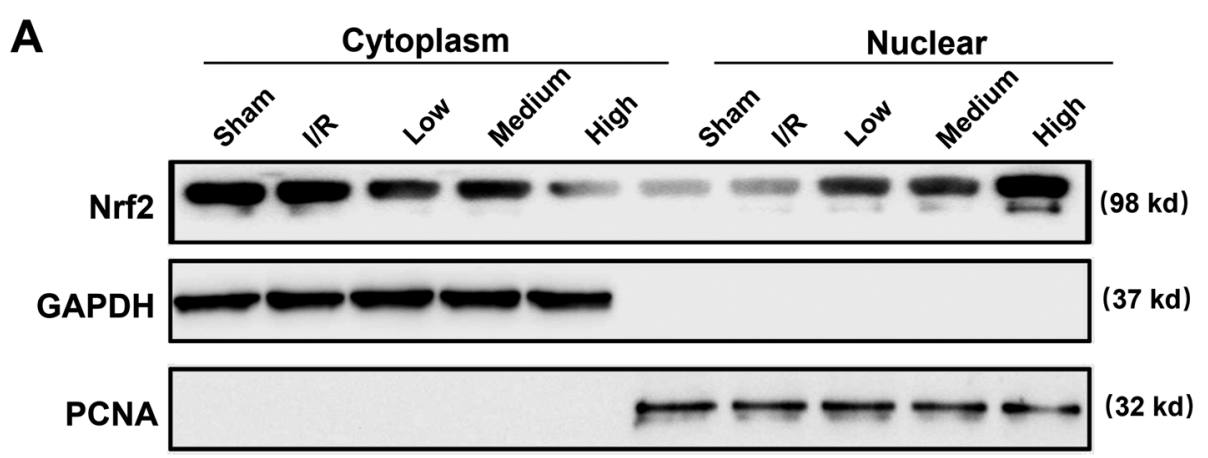

B

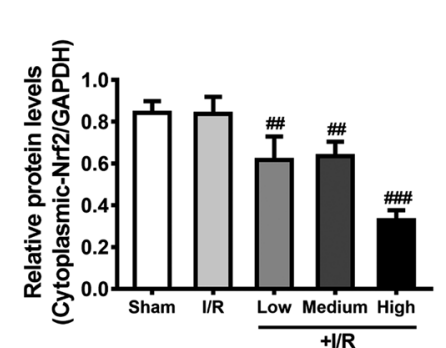

C

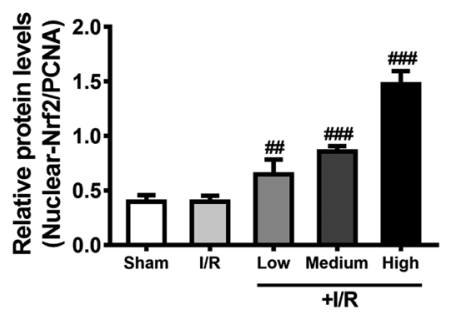

D

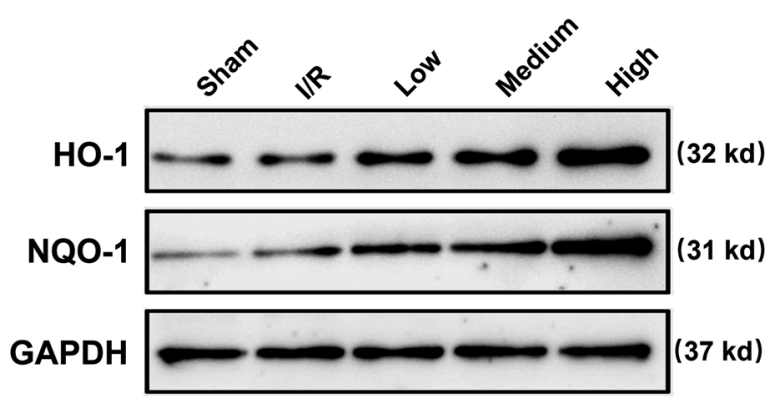

E

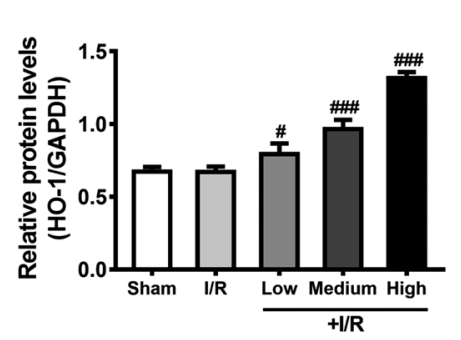

G

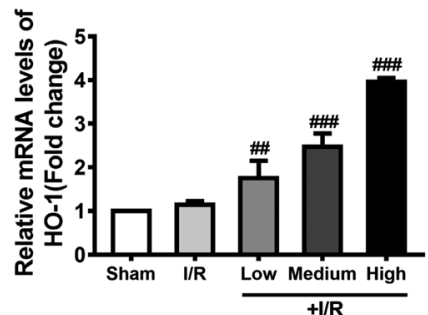

F

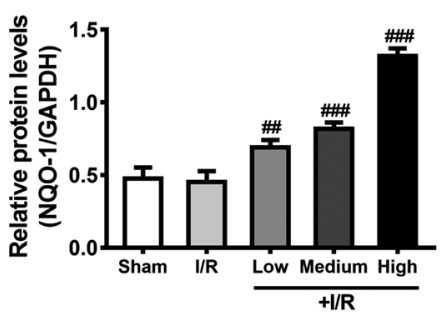

H

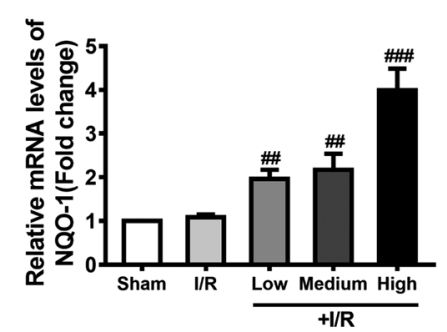

Fig. 4. Leonurusine Promoted the Nuclear Transport of Nrf2 and Expression of HO-1 and NQO-1 in Kidney Tissues

(A) Representative Western blot images of cytoplasmic and nuclear Nrf2 expression. GAPDH and PCNA were used as a loading control. (B) Quantitative protein ratio of cytoplasmic Nrf2/GAPDH. (C) Quantitative protein ratio of nuclear Nrf2/PCNA. (D) Representative Western blot images showing the expression of HO-1 and NQO-1 in the kidney tissues. Quantitative protein analysis of HO-1 (E), NQO-1 (F) expression. The mRNA levels of HO-1 (G), NQO-1 (H) in kidney tissues from sham, I/R, or LEO-pretreated rats were measured using quantitative real-time PCR. Data from three independent experiments were produced as mean \pm S.D. ${ }^{\#} p<0.05$, ${ }^{\# \# ~} p<0.01$, and \#\#\# $p<0.001$ versus $\mathrm{I} / \mathrm{R}$. 
LEO Promoted the Nuclear Transport of Nrf2 and Expression of HO-1 and NQO-1 in Kidney Tissues To explore whether LEO is intimately involved in the regulation of Nrf2 expression, the protein levels of Nrf2 in the cytoplasmic and nuclear fractions of renal tissues were detected by Western blotting. Compared with the I/R group, the LEO groups showed that nuclear translocation of Nrf2 was increased and the level of cytoplasmic Nrf2 decreased (Figs. 4A-4C). In addition, we had also observed that total Nrf2 levels of both HO-1 and NQO-1 were increased against the I/R group (Figs. $4 \mathrm{D}-4 \mathrm{~F})$. All these trends were more obvious in the high-dose LEO group and were confirmed by mRNA expression levels (Figs. 4G, 4H). These results show that LEO-induced Nrf2 increases the expression of HO-1 and NQO-1 in order to resist oxidative stress injury.

LEO Suppressed the TLR4/NF- $\kappa$ B Signal Pathway in Kidney Tissues of Ischemic AKI Rats The TLR $4 / \mathrm{NF}-\kappa \mathrm{B}$ signaling pathway is crucial to the regulation of responses, in which activated TLR4 results in an increase in the activation of the downstream factor, NF- $\kappa \mathrm{B}$, promoting cytokine production. To further estimate the reason why LEO suppressed the I/R-induced production of inflammatory cytokines, we detected the expression of several key factors in the signaling pathway. As the result shown in (Figs. 5A-5E), the protein expression of TLR4, MyD88, p-NF- $\kappa \mathrm{B}$, and $\mathrm{p}-\mathrm{I} \kappa \mathrm{B} \alpha$ was increased in the $\mathrm{I} / \mathrm{R}$ group compared to the sham group. In contrast, LEO down-regulated the protein expression of TLR4, MyD88, $\mathrm{p}-\mathrm{NF}-\kappa \mathrm{B}$, and $\mathrm{p}-\mathrm{I} \kappa \mathrm{B} \alpha$. To further confirm the effect of LEO on the activation of NF- $\kappa \mathrm{B}$, the nuclear translocation of NF- $\kappa \mathrm{B}$ was detected by Western blot. Compared with the I/R group, the LEO groups showed that nuclear translocation of NF- $\kappa \mathrm{B}$ was decreased (Figs. $5 \mathrm{~F}-5 \mathrm{H}$ ). These results suggest that LEO may have a protective effect on rats regarding $\mathrm{I} / \mathrm{R}$-induced AKI by suppressing the TLR4/NF- $\kappa$ B signaling pathway.

\section{Discussion}

Acute kidney injury (AKI) is a common clinical problem, with a high morbidity. There is no targeted pharmacologic therapy is effective in patients with severe I/R-induced AKI. It is imperative to figure out new therapeutic strategies. This study is mainly divided into two parts. The first is to establish an $\mathrm{I} / \mathrm{R}$ rat model to evaluate the effects of LEO pretreatment. The second part is to explore whether Nrf2-regulated antioxidant defense and TLR4/NF- $\kappa \mathrm{B}$ pathway mediated the production of inflammatory cytokines is involved in the above process.

As mentioned earlier, LEO is a special monomer with biological activity that exists in traditional Chinese medicine and is widely used in clinical gynecology in China. In addition, LEO has been shown to inhibit the production of ROS to attenuate LPS-induced acute kidney injury. ${ }^{19)}$ Recently, LEO has a protective effect on kidney damage in adenineinduced chronic renal failure rat model and LEO reduces the serum levels of BUN and CRE, which indicates that LEO can be used as a further potential therapeutic drug for AKI. ${ }^{14,20)}$ Besides, the dosage of LEO used in the experiment referred to previous literature reports. ${ }^{20,21)} \mathrm{Scr}$ and BUN are both commonly renal function parameters. The values of Scr and BUN in the serum of I/R models were detected at the starting of this research. In present study, we found that I/R injury caused an increase of Scr and BUN and different dose of LEO stimulation significantly reduced the content of Scr and BUN in the serum of $\mathrm{I} / \mathrm{R}$ rat. The decrease of the concentration of Scr and BUN indicated that the filtration function of rat glomerulus has been repaired. Additionally, based on pathologic score, we further found that LEO also weakened the kidney damage in the I/R rat model. All together, these data demonstrates that LEO prevented renal dysfunction and histologic damage in ischemic AKI.

There is increasing evidence suggesting that oxidative stress and inflammation are strongly linked to the pathogenesis of $\mathrm{I} / \mathrm{R}$ injury. Besides, oxidative stress and even lipid peroxidation are prone to occur in kidney tissues with $\mathrm{I} / \mathrm{R}$ damage. By detecting iconic indicators of oxidative stress, we knew that $\mathrm{I} / \mathrm{R}$ injury not only caused the decrease of the antioxidantrelated factors (SOD, GSH, and CAT), but also led to the increase of the oxidant-related factors (MDA). Our findings are similar to the previous research performed by Chen et al. ${ }^{22}$ ) Moreover, compared with I/R group, pretreating with three different doses of LEO significantly reduced the level of MDA and promoted the activity of SOD, CAT, and GSH. To demonstrate the relationship between excessive inflammation responses and the pathological process of AKI, we measured the levels of inflammatory cytokines in serum. Our ELISA results showed the suppressing action of LEO on the levels of inflammatory cytokines in the serum of $\mathrm{I} / \mathrm{R}$ model rats, including IL- $1 \beta$, TNF- $\alpha$, IL- 6 , and IL- 8 . The preceding findings suggest that LEO preconditioning alleviates the production of inflammatory cytokines in rats with $\mathrm{I} / \mathrm{R}$ injury.

The mechanism of LEO pretreatment reducing renal oxidative stress and inflammation to recover the I/R injury remains unclear. Previous studies support that component of the Nrf2 pathway are important sites to investigate oxidative stress. The Nrf2 pathway regulates endogenous antioxidant defense resistance to reduce the oxidative damage in the body. ${ }^{14,21)}$ In fact, it has been proven that under normal conditions, Nrf2 binds to Keap1 cysteines to anchor in the cytoplasm. ${ }^{23)}$ When an exception occurs to activate oxidative stress, the Nrf2-Keap1 complex will rapidly separate and free Nrf2 translocate into the nucleus. The Nrf2 in the nucleus actives antioxidant enzymes, including HO-1 and NQO-1, which inhibits the oxidative stress. Based on our Western blot and real-time PCR data, LEO decreased the Nrf2 protein expression in the cytoplasm and increased accumulate in the nucleus of rats with I/R damage. Moreover, we found high expression of both HO-1 and NQO-1. This result not only implies that LEO increased the nuclear transposition of Nrf2, but also reveals downstream antioxidant enzymes, HO-1 and NQO-1 are important to keep the body in a balanced state of the oxidant-antioxidant. ${ }^{24)}$

The TLR4/NF- $\kappa \mathrm{B}$ signaling pathway is involved in the inflammatory response caused by a variety of pathological processes. It has been reported that the TLR $4 / \mathrm{NF}-\kappa \mathrm{B}$ plays a vital role in the disease progression of renal inflammation response and fibrosis. ${ }^{25)}$ Normally, TLR4 is broadly expressed in healthy renal tissues but at a lower level. When the kidney is infected or damaged, the expression of TLR4 increases. Some studies have observed the effect of I/R injury on the expression level of TLR4, and the results show that after injury, the level of TLR4 in kidney tissue increases. ${ }^{26)}$ Activated of TLR4 regulates the downstream mediators MyD88 and $\mathrm{NF}-\kappa \mathrm{B}$, while $\mathrm{p}-\mathrm{I} \kappa \mathrm{B} \alpha$ can promote the phosphorylation of inactive $\mathrm{NF}-\kappa \mathrm{B}$ in the cytoplasm and enter the nucleus. Our study 
A

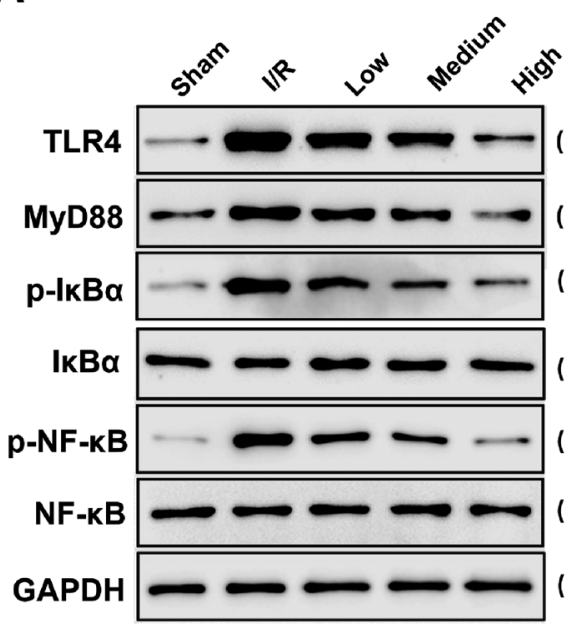

D

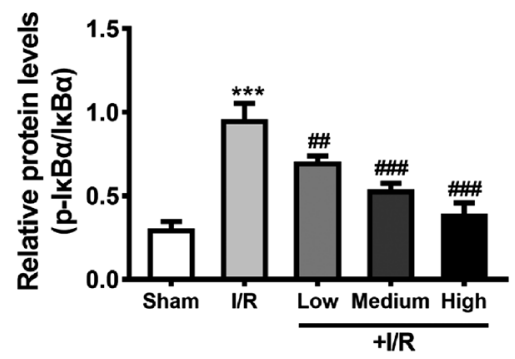

B

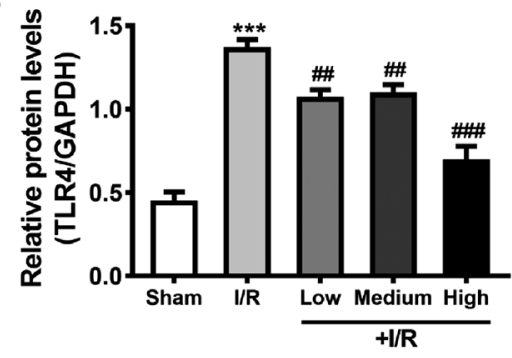

C

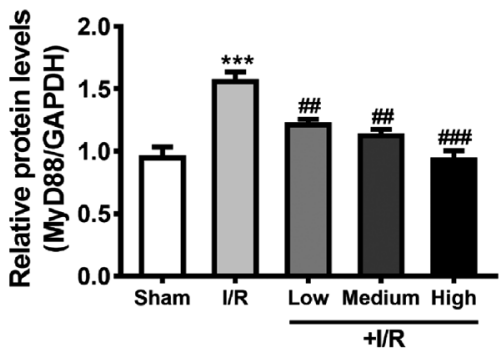

E

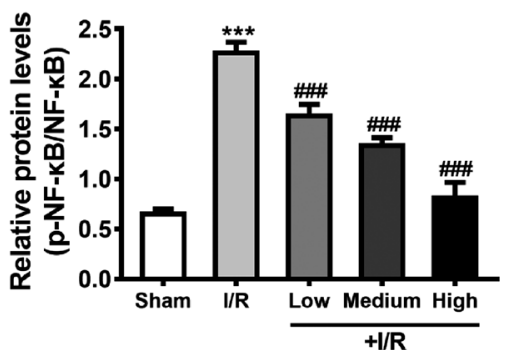

F

Cytoplasm

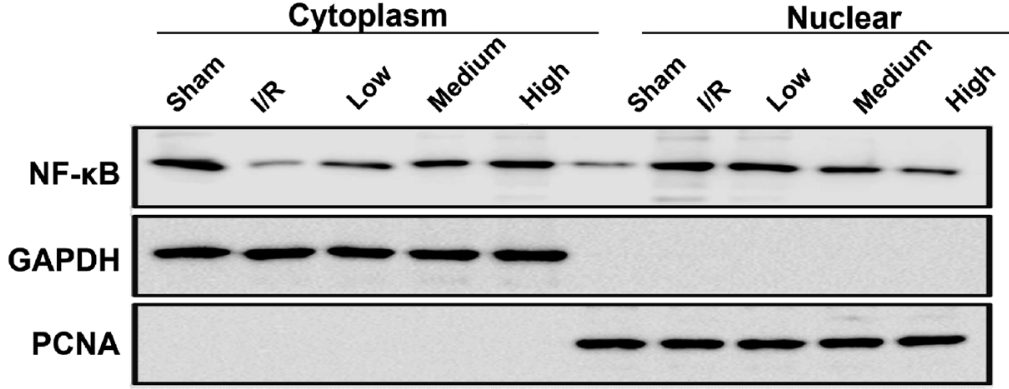

(65 kd)

$(37 \mathrm{kd})$

G

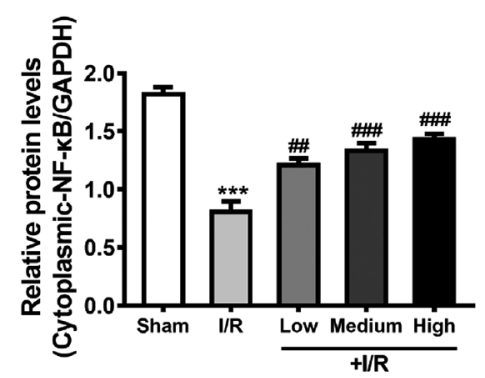

H

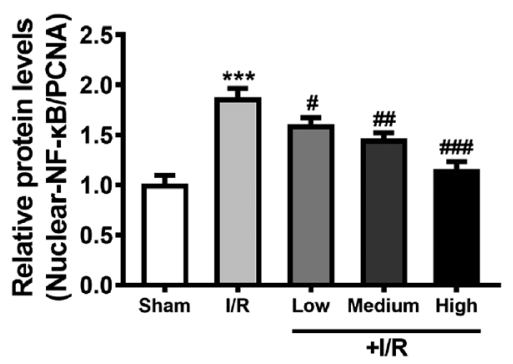

Fig. 5. LEO Suppressed the TLR4/NF- $\kappa \mathrm{B}$ Signal Pathway in Kidney Tissues of Ischemic AKI Rats

(A) Representative Western blot images of TLR4, MyD88, p-I $\kappa \mathrm{B} \alpha, \mathrm{I} \kappa \mathrm{B} \alpha, \mathrm{p}-\mathrm{NF}-\kappa \mathrm{B}$, and NF- $\kappa \mathrm{B}$ expression. Quantitative protein ratio of TLR4/GAPDH (B), MyD88/ GAPDH (C), $\mathrm{p}-\mathrm{I} \kappa \mathrm{B} \alpha / \mathrm{I} \kappa \mathrm{B} \alpha(\mathrm{D})$, and $\mathrm{p}-\mathrm{NF}-\kappa \mathrm{B} / \mathrm{NF}-\kappa \mathrm{B}$ (E). (F) Representative Western blot images of cytoplasmic and nuclear NF- $\kappa \mathrm{B}$ expression. GAPDH and PCNA were used as a loading control. (G) Quantitative protein ratio of cytoplasmic NF- $\kappa$ B/GAPDH. (H) Quantitative protein ratio of nuclear NF- $\kappa$ B/PCNA. Data from three independent experiments were produced as mean \pm S.D. ${ }^{* * *} p<0.001$ versus sham; ${ }^{\#} p<0.05,{ }^{\# \#} p<0.01$, and ${ }^{\# \# \#} p<0.001$ versus I/R.

found that compared with the sham group, the contents of TLR4, MyD88, $\mathrm{p}-\mathrm{NF}-\kappa \mathrm{B}$, and $\mathrm{p}-\mathrm{I} \kappa \mathrm{B} \alpha$ in the kidney tissue of the I/R group increased. Moreover, LEO pretreatment downregulated the protein expression of TLR4, MyD88, p-NF- $\kappa \mathrm{B}$, and $\mathrm{p}-\mathrm{I} \kappa \mathrm{B} \alpha$. These outcomes suggest that LEO has a positive effect on rats regarding I/R-induced AKI by suppressing the

\section{TLR4/NF- $\kappa \mathrm{B}$ signaling pathway.}

In conclusion, we demonstrate that LEO pretreatment has a protective effect to prevent ischemic AKI through activation of Nrf2 nuclear translocation resisting oxidative stress injury and inhibition of the TLR4/NF- $\kappa \mathrm{B}$ pathway mediated inflammatory gene expression. Our findings suggest that Leonurine 
may be used as a new natural product for the treatment of AKI.

Conflict of Interest The authors declare no conflict of interest.

\section{References}

1) Scholz H., Boivin F. J., Schmidt-Ott K. M., Bachmann S., Eckardt K. U., Scholl U. I., Persson P. B., Nat. Rev. Nephrol., 17, 335-349 $(2021)$.

2) Ucar B. I., Erikci A., Kosemehmetoglu K., Ozkul C., Iskit A. B., Ucar G., Zeren S., Int. J. Surg., 83, 89-97 (2020).

3) Ronco C., Ricci Z., De Backer D., Kellum J. A., Taccone F. S., Joannidis M., Pickkers P., Cantaluppi V., Turani F., Saudan P., Bellomo R., Joannes-Boyau O., Antonelli M., Payen D., Prowle J. R., Vincent J. L., Crit. Care, 19, 146 (2015).

4) Rabb H., Griffin M. D., McKay D. B., Swaminathan S., Pickkers P., Rosner M. H., Kellum J. A., Ronco C., J. Am. Soc. Nephrol., 27, 371-379 (2016)

5) Tomsa A., Alexa A., Junie M., Rachisan A., Ciumarnean L. J., PeerJ, 7, e8046 (2019).

6) Nezu M., Suzuki N., Int. J. Mol. Sci., 21, 2951 (2020).

7) Ge M., Yao W., Yuan D., Zhou S., Chen X., Zhang Y., Li H., Xia Z., Hei Z. J., Cell Death Dis., 8, e2841 (2017).

8) Sun Y., Yang T., Leak R. K., Chen J., Zhang F., CNS Neurol. Disord. Drug Targets, 16, 326-338 (2017).

9) Vázquez-Carballo C., Guerrero-Hue M., García-Caballero C., Rayego-Mateos S., Opazo-Ríos L., Morgado-Pascual J., HerenciaBellido C., Vallejo-Mudarra M., Cortegano I., Gaspar M., de Andrés B., Egido J., Moreno J. J., Int. J. Mol. Sci., 22, 816 (2021).

10) Loh K. P., Qi J., Tan B. K., Liu X. H., Wei B. G., Zhu Y. Z., Stroke,
41, 2661-2668 (2010).

11) Huang L., Xu D. Q., Chen Y. Y., Yue S. J., Tang Y. P., Brain Behav, 11, e01995 (2021)

12) Sun J., Huang S. H., Zhu Y. C., Whiteman M., Wang M. J., Tan B. K., Zhu Y. Z., Life Sci., 76, 3043-3056 (2005).

13) Song X., Wang T., Zhang Z., Jiang H., Wang W., Cao Y., Zhang N., Inflammation, 38, 79-88 (2015).

14) Chen P., Chen F., Zhou B. H., Aging, 11, 7339-7356 (2019).

15) Ozturk H., Cetinkaya A., Duzcu S. E., Tekce B. K., Ozturk H., Biomed. Pharmacother., 98, 656-661 (2018).

16) Xu L., Jiang X., Wei F., Zhu H., Mol. Med. Rep., 18, 1582-1590 (2018).

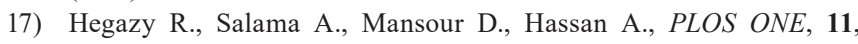
$\mathrm{e} 0151486$ (2016).

18) Dennis J. M., Witting P. K., Nutrients, 9, 718 (2017).

19) Xu D., Chen M., Ren X., Ren X., Wu Y. J., Fitoterapia, 97, 148-155 (2014).

20) Bai W., Wang S., An S., Guo M., Gong G., Liu W., Ma S., Li X., Fu J., Yao W., Oncotarget, 9, 15498-15511 (2018).

21) Xie Y. Z., Zhang X. J., Zhang C., Yang Y., He J. N., Chen Y. X., CNS Neurosci. Ther., 25, 1006-1017 (2019).

22) Chen M., Yan X. T., Ye L., Tang J. J., Zhang Z. Z., He X. H., Oxid. Med. Cell. Longev., 2020, 6120194 (2020).

23) Bellezza I., Giambanco I., Minelli A., Donato R., Biochim. Biophys. Acta Mol. Cell. Res., 1865, 721-733 (2018).

24) Wang X., Hai C. X., Liang X., Yu S. X., Zhang W., Li Y. L., J. Ethnopharmacol., 127, 424-432 (2010).

25) Yang L., Liu G., Lian K., Qiao Y., Zhang B., Zhu X., Luo Y., Shang Y., Gu X. J., J. Anim. Sci., 97, 1679-1692 (2019).

26) Zhang X., Du Q., Yang Y., Wang J., Dou S., Liu C., Duan J., Biomed. Pharmacother., 91, 1042-1052 (2017). 